\title{
On the head and the tail of the colored Jones polynomial
}

\author{
Oliver T. Dasbach and Xiao-Song Lin
}

\begin{abstract}
The colored Jones polynomial is a function $J_{K}: \mathbb{N} \longrightarrow \mathbb{Z}\left[t, t^{-1}\right]$ associated with a knot $K$ in 3-space. We will show that for an alternating knot $K$ the absolute values of the first and the last three leading coefficients of $J_{K}(n)$ are independent of $n$ when $n$ is sufficiently large. Computation of sample knots indicates that this should be true for any fixed leading coefficient of the colored Jones polynomial for alternating knots. As a corollary we get a volume-ish theorem for the colored Jones polynomial.
\end{abstract}

\section{Introduction}

The celebrated volume conjecture of Kashaev [Kas97] and Murakami and Murakami [MM01] claims that the colored Jones polynomial determines the volume of a hyperbolic knot complement. This conjecture is wide open. Recall that the colored Jones polynomial of a knot $K$ is a sequence of one variable Laurent polynomials $J_{K}(n)$ indexed by a positive integer $n$, the color. For $n=2$, the normalized version of $J_{K}(n), J_{K}^{\prime}(n)$, it is the classical Jones polynomial.

By using results of Lackenby, Agol and Thurston [Lac04] we showed in [DL04] that there is a volume-ish theorem for the Jones polynomial of alternating knots: the volume of a hyperbolic alternating knot is linearly bounded by the sum of the absolute values of the second leading and the second lowest coefficient of the Jones polynomial $J_{K}^{\prime}(2)$.

Here we will extend our results to the colored Jones polynomial of alternating knots. We will show that in this case the colored Jones polynomial has a well defined head and tail: the leading three and the lowest three coefficients are independent of the color, for $n \geqslant 3$. It turns out that these coefficients have a particularly nice form and can easily be computed. It is interesting to note, though, that the third coefficient of the colored Jones polynomial $J_{K}^{\prime}(3)$ is in general not determined by the Jones polynomial $J_{K}^{\prime}(2)$.

Moreover, the coefficients that gave rise to the volume-ish theorem for the Jones polynomial persists in all the colored Jones polynomials: the second leading coefficient and the second lowest coefficient are independent of the color. As a corollary we will get a volume-ish theorem for the colored Jones polynomial, independent of the color $n$. Thus there is indeed a deeper connection between the colored Jones polynomial and the volume of hyperbolic knots, at least for alternating knots.

Received 21 December 2005, accepted in final form 4 April 2006.

2000 Mathematics Subject Classification 57M25.

Keywords: colored Jones polynomial, volume-ish theorem, volume conjecture.

The first author was supported in part by NSF grants DMS-0306774 and DMS-0456275 (FRG). The second author was supported in part by NSF grants DMS-0404511 and DMS-0456217 (FRG).

This journal is (C) Foundation Compositio Mathematica 2006. 


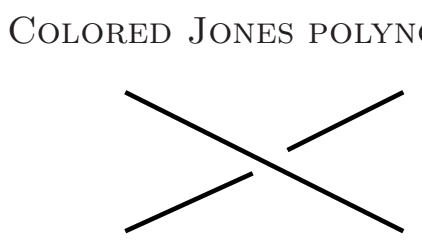

A crossing in $D$

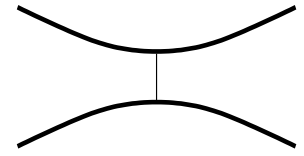

A-splicing

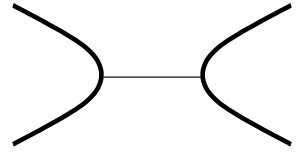

B-splicing

Figure 1 . Splicings of a crossing, $A$-graph and $B$-graph.

\section{Background}

Let $D$ be a knot diagram. For each crossing of $D$, we may apply two kinds of splicings (see Figure 1 ) as in the Kauffman skein relation. One splicing is called $A$-splicing and the other $B$-splicing. A state $s$ of $D$ is a choice of $A$-splicing or $B$-splicing for each crossing of $D$. After applying a state $s$ to splice each crossing of $D$, we change $D$ to a diagram of a collection of disjoint simple closed curves (circles) in the plane. Let $d(s)$ be the number of the resulting circles. Also let $\alpha(s)$ (respectively $\beta(s)$ ) be the number of $A$-splicings (respectively $B$-splicings) in the state $s$. With these notations, the Kauffman bracket of $D$ is

$$
\langle D\rangle=\sum_{s} A^{\alpha(s)-\beta(s)}\left(-A^{2}-A^{-2}\right)^{d(s)-1},
$$

where the sum is over all states $s$ of $D$.

Definition 2.1. The $A$-graph $A(D)$ of a knot diagram $D$ has its set of vertices equal to the set of circles in the all $A$-splicing state, and there is an edge joining two vertices of $A(D)$ for each crossing in $D$ between the corresponding circles. Similarly, we define the $B$-graph $B(D)$ of $D$ using the all $B$-splicing state.

To the best of our knowledge, the notion of $A$-graph and $B$-graph of a knot diagram $D$ was first introduced by A. Stoimenow ([Sto04], compare with [Thi88]).

Definition 2.2. A knot diagram $D$ is called $A$-adequate (respectively $B$-adequate) if there are no loops, i.e. edges that start and terminate at the same vertex, in the $A$-graph $A(D)$ (respectively the $B$-graph $B(D)$ ). A knot diagram $D$ is called adequate if it is both $A$-adequate and $B$-adequate.

It is easy to see that a reduced alternating knot diagram is adequate. In fact, in this case the $A$ - and the $B$-graph are the two checkerboard graphs of the knot diagram.

Remark 2.3. A diagram $D$ of a knot is $A$-adequate if and only if the corresponding diagram $D^{*}$ of its mirror image is $B$-adequate. Furthermore, the Kauffman bracket of the mirror image $D^{*}$ equals the Kauffman bracket of $D$ after substituting $A^{*}$ for the variable $A$. Therefore, all theorems on $A$-adequate knots have a corresponding formulation for $B$-adequate knots. In the sequel we will frequently omit this formulation to make the theorems more readable. 


\section{O. T. DAsbach And X.-S. Lin}

The reduced $A$-graph $A(D)^{\prime}$ of a knot diagram is obtained from the $A$-graph $A(D)$ by keeping the same set of vertices but reducing all multiple edges to one for each pair of vertices. So a knot diagram $D$ is $A$-adequate if and only if $A(D)^{\prime}$ is a simple graph, i.e. does not have loops or multiple edges. Similarly, we have the reduced $B$-graph $B(D)^{\prime}$ and $D$ is $B$-adequate if and only if $B(D)^{\prime}$ is a simple graph.

Let $D$ be a knot diagram of $c=c(D)$ crossings. The number of vertices of $A(D)^{\prime}$, which equals the number of vertices in the unreduced graph $A(D)$, is denoted by $v=v(D)$ and the number of edges of $A(D)^{\prime}$ by $e=e(D)$.

The following theorem is a generalization of our corresponding result in [DL04] that we showed for alternating knots.

Theorem 2.4 (Stoimenow). Suppose $D$ is A-adequate. Then we have

$$
\langle D\rangle=(-1)^{v-1} A^{c+2 v-2}+(-1)^{v-2}(e-v+1) A^{c+2 v-6}+\text { lower order terms. }
$$

Proof. Recall that $e=e(D)$ is the number of edges of the reduced graph $A(D)^{\prime}$. Let $k_{i}$ be the multiplicity of the $i$ th edge of $A(D)^{\prime}$ in $A(D)$, for $i=1,2, \ldots, e$.

For a state $s$ of $D$ where there are at least two $B$-splicings appearing at edges of $A(D)$ which reduce to different edges in $A(D)^{\prime}$, its contribution to $\langle D\rangle$ is

$$
A^{c-2 \beta(s)}\left(-A^{2}-A^{-2}\right)^{d(s)-1}=(-1)^{d(s)-1} A^{c-2 \beta(s)+2 d(s)-2}+\text { lower degree terms, }
$$

where $d(s) \leqslant v-2+\beta(s)-2$. Now

$$
c-2 \beta(s)+2 d(s)-2 \leqslant c+2 v-10 .
$$

So we only need to consider states whose $B$-splicings all appear on the same $k_{i}$ multiple edges of $A(D)$. The contribution of such states in $\langle D\rangle$ is

$$
\begin{aligned}
A^{c}(- & \left.A^{2}-A^{-2}\right)^{v-1}+\sum_{i=1}^{e} \sum_{j=1}^{k_{i}}\left(\begin{array}{c}
k_{i} \\
j
\end{array}\right) A^{c-2 j}\left(-A^{2}-A^{-2}\right)^{v-2+j-1} \\
= & (-1)^{v-1}\left(A^{c+2 v-2}+(v-1) A^{c+2 v-6}+\cdots\right) \\
& +\sum_{i=1}^{e} \sum_{j=1}^{k_{i}}\left(\begin{array}{c}
k_{i} \\
j
\end{array}\right)(-1)^{v+j-3} A^{c+2 v-6}+\cdots \\
= & (-1)^{v-1} A^{c+2 v-2}+(-1)^{v-2}(e-v+1) A^{c+2 v-6}+\text { lower degree terms. }
\end{aligned}
$$

This proves the theorem.

Note that when the graph $A(D)^{\prime}$ is connected, we have

$$
\left.e-v+1=\beta_{1}\left(A(D)^{\prime}\right) \quad \text { (the first Betti number of } A(D)^{\prime}\right) .
$$

This will be important to us.

Lemma 2.5. Let $D$ be an $A$-adequate (respectively $B$-adequate) knot diagram and $D^{n}$ be its blackboard $n$ cabling. Then $D^{n}$ is $A$-adequate (respectively $B$-adequate). Furthermore, we have

$$
\beta_{1}\left(A\left(D^{n}\right)^{\prime}\right)=\beta_{1}\left(A(D)^{\prime}\right) .
$$

Proof. The first claim was shown by Lickorish (see e.g. [Lic97]). Figure 2 depicts the $A$-splicing of the blackboard cabling of a knot diagram $D$.

If $D$ is $A$-adequate, then $A(D)^{\prime}$ is a simple graph. For a vertex $V$ of $A(D)^{\prime}$, let the edges coming out of $V$ be $E_{1}, E_{2}, \ldots, E_{k}$. Then in $A\left(D^{n}\right)^{\prime}$, we have $n$ vertices associated with $V$, i.e. $V_{1}, V_{2}, \ldots, V_{n}$, and $n-1$ edges, i.e. $V_{1} V_{2}, V_{2} V_{3}, \ldots, V_{n-1} V_{n}$. The edges $E_{1}, E_{2}, \ldots, E_{k}$ of $A(D)^{\prime}$ are still in $A\left(D^{n}\right)^{\prime}$ 


\section{COLORED JONES POLYNOMial}

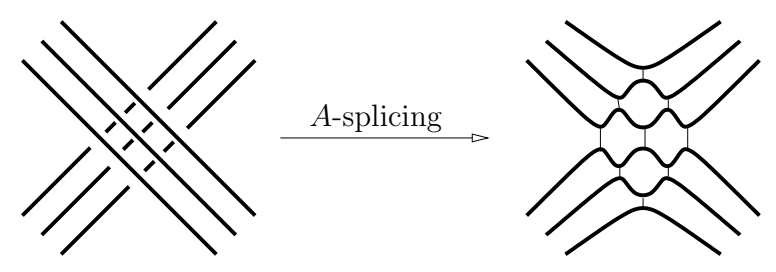

Figure 2. $A$-splicing of the cabling of a crossing.

as edges coming out of $V_{1}$. Thus, $A\left(D^{n}\right)^{\prime}$ is equal to $A(D)^{\prime}$ with a chain $V_{1} V_{2} \cup V_{2} V_{3} \cup \cdots \cup V_{n-1} V_{n}$ attached to each vertex $V=V_{1}$. So $A\left(D^{n}\right)^{\prime}$ is still a simple graph and $D^{n}$ is $A$-adequate.

The second claim follows easily from the structure of $A\left(D^{n}\right)^{\prime}$ described above.

\section{The colored Jones polynomial}

We will use the Chebyshev basis of the Kauffman bracket skein module of $S^{1} \times[0,1]$ to express the colored Jones polynomial; see e.g. [Lic97].

Let $S_{n}(x), n \geqslant 0$, be polynomials of $x$ specified by the following recurrence relation and initial values:

$$
S_{n+1}=x S_{n}-S_{n-1}, \quad S_{0}(x)=1, \quad S_{1}(x)=x .
$$

Inductively, we have

$$
S_{n}(x)=x^{n}+(1-n) x^{n-2}+\text { lower degree terms. }
$$

Suppose that $D$ is a knot diagram of a knot $K$. Let $J_{K}(n)$ be the colored Jones polynomial of $K$ corresponding to the $n$-dimensional irreducible $U_{q}\left(\mathfrak{s l}_{2}\right)$ module. Here, $J_{K}(2)$ is the classical Jones polynomial multiplied by $\left(A^{2}+A^{-2}\right)$. We have

$$
J_{K}(n+1)=\left[(-1)^{n} A^{n^{2}+2 n}\right]^{-w(D)}(-1)^{n-1}[2]\left\langle S_{n}(D)\right\rangle,
$$

where $[2]=A^{2}+A^{-2}, w(D)$ is the writhe of $D$, and $S_{n}(D)$ is a linear combination of blackboard cablings of $D$ obtained using the Chebyshev polynomial $S_{n}(x)$. By Equation (1), we have

$$
S_{n}(D)=D^{n}+(1-n) D^{n-2}+\text { lower degree cablings of } D \text {. }
$$

For the unknot diagram $O$, we have

$$
J_{O}(n)=\frac{A^{2 n}-A^{-2 n}}{A^{2}-A^{-2}}=A^{2 n-2}+A^{2 n-6}+\cdots+A^{-2 n+6}+A^{-2 n+2}:=[n] .
$$

The normalized colored Jones polynomial is

$$
J_{K}^{\prime}(n)=J_{K}(n) /[n]
$$

and $J_{K}^{\prime}(2)$ is the classical Jones polynomial.

We are interested in the leading coefficients of $J_{D}^{\prime}(n)$. We write

$$
J_{D}^{\prime}(n)=a A^{k}+b A^{k-4}+c A^{k-8}+\cdots,
$$

thus

$$
\begin{aligned}
J_{D}(n) & =J_{D}^{\prime}(n)[n] \\
& =\left(a A^{k}+b A^{k-4}+c A^{k-8}+\cdots\right)\left(A^{2 n-2}+A^{2 n-6}+\cdots\right) \\
& =a A^{k+2 n-2}+(a+b) A^{k+2 n-6}+(a+b+c) A^{k+2 n-10}+\cdots
\end{aligned}
$$




\section{O. T. DASBACH AND X.-S. LiN}

Suppose now that $D$ is an $A$-adequate knot diagram. Since all blackboard cablings of $D$ are adequate, using Theorem 2.4, Lemma 2.5, and Equation (2), we have

$$
\begin{aligned}
\left\langle S_{n}(D)\right\rangle= & \left\langle D^{n}\right\rangle+(1-n)\left\langle D^{n-2}\right\rangle+\cdots \\
= & (-1)^{n v-1} A^{n^{2} c+2 n v-2}+(-1)^{n v-2} \beta_{1}\left(A(D)^{\prime}\right) A^{n^{2} c+2 n v-6}+\cdots \\
& +(-1)^{(n-2) v-1}(1-n) A^{(n-2)^{2} c+2(n-2) v-2} \\
& +(-1)^{(n-2) v-2}(1-n) \beta_{1}\left(A(D)^{\prime}\right) A^{(n-2)^{2} c+2(n-2) v-6}+\cdots
\end{aligned}
$$

Compare the second highest degree of $\left\langle D^{n}\right\rangle$ and the highest degree of $\left\langle D^{n-2}\right\rangle$ :

$$
\left(n^{2} c+2 n v-6\right)-\left((n-2)^{2} c+2(n-2) v-2\right)=(4 n-4) c+4 v-4>0 .
$$

So,

$$
\left\langle S_{n}(D)\right\rangle=(-1)^{n v-1} A^{n^{2} c+2 n v-2}+(-1)^{n v-2} \beta_{1}\left(A(D)^{\prime}\right) A^{n^{2} c+2 n v-6}+\text { lower degree terms. }
$$

Finally, we have

$$
\begin{aligned}
{[2]\left\langle S_{n}(D)\right\rangle=} & \left(A^{2}+A^{-2}\right)\left\langle S_{n}(D)\right\rangle \\
= & (-1)^{n v-1} A^{n^{2} c+2 n v}+\left((-1)^{n v-1}+(-1)^{n v-2} \beta_{1}\left(A(D)^{\prime}\right)\right) A^{n^{2} c+2 n v-4} \\
& + \text { lower degree terms. }
\end{aligned}
$$

Theorem 3.1. Let $D$ be an $A$-adequate knot diagram. Write

$$
J_{D}^{\prime}(n)=a_{n} A^{k_{n}}+b_{n} A^{k_{n}-4}+\text { lower degree terms. }
$$

Then, we have

$$
\left|a_{n}\right|=1 \quad \text { and } \quad\left|b_{n}\right|=\beta_{1}\left(A(D)^{\prime}\right) .
$$

Proof. This follows directly from the previous calculations.

Remark 3.2. Note that the difference between highest degrees of $\left\langle D^{n}\right\rangle$ and $\left\langle D^{n-2}\right\rangle$, respectively, is $O(n)$. So when $n$ is getting large, any fixed leading portion of coefficients of $J_{K}^{\prime}(n)$ will only depend on the coefficients of $\left\langle D^{n}\right\rangle$. For the first and second leading coefficients of $\left\langle D^{n}\right\rangle$, we have shown that they depend only on $n$ by a sign when $K$ has an $A$-adequate diagram. We consider the third leading coefficient of $\left\langle D^{n}\right\rangle$ in the next section.

\section{The third coefficient of $J_{K}^{\prime}(n)$}

The difficulties in computing the third leading coefficient are that it does not depend on the topological type of the $A$-graph and its reduced graph any more. Informations on the embedding of the $A$-graph are needed.

We first will extend Theorem 2.4. Suppose that a knot diagram $D$ is $A$-adequate. The vertices of $A(D)$ are represented by disjoint circles in the plane. Let $E$ and $E^{\prime}$ be two different edges in $A(D)^{\prime}$. We say that they are disjoint if $E \cap E^{\prime}=\emptyset$. If $E$ and $E^{\prime}$ share a common vertex $V$, then there are two possibilities. In one case, the circle representing $V$ can be divided into two semi-circles such that vertices of multiple edges $E$ and $E^{\prime}$ lie on different semi-circles respectively. We say that $E$ and $E^{\prime}$ are separated at $V$ in this case. Otherwise, we say that $E$ and $E^{\prime}$ are mixed at $V$. See Figure 3 , in which the pairs $\left\{E_{1}, E_{4}\right\}$ are $\left\{E_{1}, E_{5}\right\}$ are mixed, and the pairs $\left\{E_{4}, E_{5}\right\}$ and $\left\{E_{4}, E_{6}\right\}$ are separated.

Suppose $E$ and $E^{\prime}$ are two edges of $A(D)^{\prime}$ mixed at a vertex $V$. Then in $A(D)$, the multiple edge $E$ (respectively $E^{\prime}$ ) is partitioned into sets of $p_{1}, p_{2}, \ldots, p_{m}$ (respectively $q_{1}, q_{2}, \ldots, q_{m}$ ) parallel multiple edges, so that the endpoints of these edges on the circle $V$ are placed alternatively 

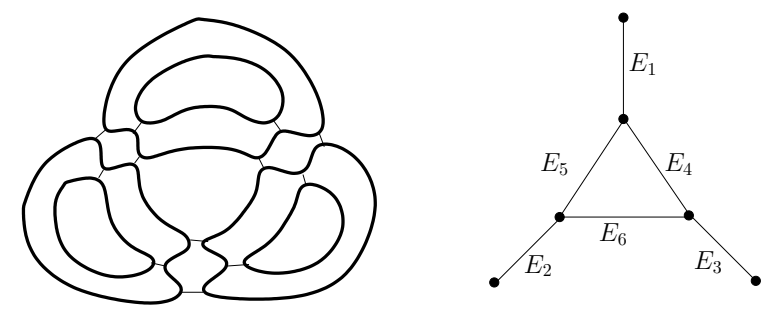

Figure 3. $A(D)$ and $A(D)^{\prime}$ where $D$ is the 2-cable of the left-trefoil diagram.

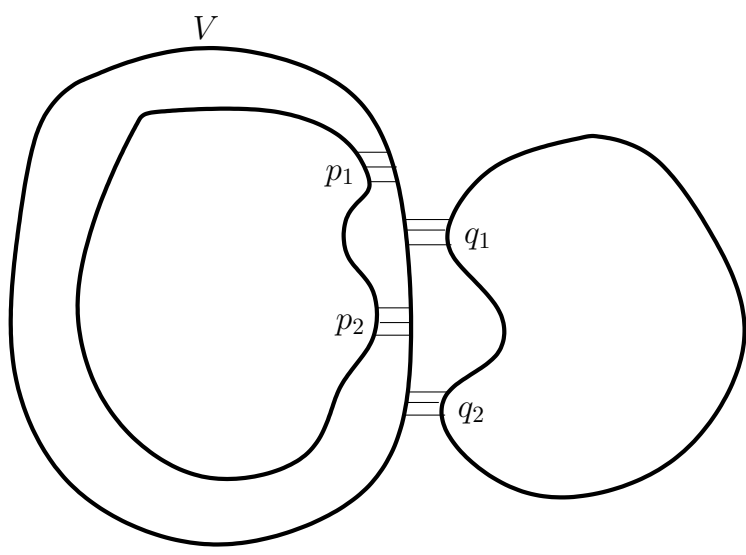

Figure 4. Two multiple edges mixed at $V$.

as $p_{1}, q_{1}, p_{2}, q_{2}, \ldots, p_{m}, q_{m}$. We must have $m \geqslant 2$ (see Figure 4 ). If $D$ is a reduced alternating knot diagram, we do not have such pairs of edges $E$ and $E^{\prime}$ in $A(D)^{\prime}$.

Theorem 4.1. Suppose $D$ is $A$-adequate. Then we have

$$
\begin{aligned}
\langle D\rangle= & (-1)^{v-1} A^{c+2 v-2}+(-1)^{v-2}(e-v+1) A^{c+2 v-6} \\
& +(-1)^{v-3}\left(\left(\begin{array}{c}
v-1 \\
2
\end{array}\right)-e(v-2)+\mu+\left(\begin{array}{l}
e \\
2
\end{array}\right)-\theta-\tau\right) A^{c+2 v-10} \\
& + \text { lower order terms, }
\end{aligned}
$$

where $\mu$ is the number of edges in $A(D)^{\prime}$ whose multiplicity in $A(D)$ is larger than $1, \theta$ is the number of pairs of edges in $A(D)^{\prime}$ mixed at a vertex, and $\tau$ is the number of triangles in $A(D)^{\prime}$.

When $D$ is a reduced alternating knot diagram, then $\theta=0$. So this theorem generalizes the formula of $\langle D\rangle$ in [DL04] for reduced alternating knot diagrams.

Proof. Let $s$ be a state of $D$ with at least three $B$-splicings such that (1) they appear at edges of $A(D)$ which reduce to three different edges in $A(D)^{\prime}$, and (2) these three edges of $A(D)^{\prime}$ do not form a triangle in $A(D)^{\prime}$. Then the contribution of $s$ to $\langle D\rangle$ is

$$
A^{c-2 \beta(s)}\left(-A^{2}-A^{-2}\right)^{d(s)-1}=(-1)^{d(s)-1} A^{c-2 \beta(s)+2 d(s)-2}+\text { lower degree terms, }
$$

where $d(s) \leqslant v-3+\beta(s)-3$. Now

$$
c-2 \beta(s)+2 d(s)-2 \leqslant c+2 v-14 .
$$

So we only need to consider the following four cases. 
Case 1. States whose $B$-splicings all appear on the same $k_{i}$ multiple edges of $A(D)$. The total contribution of such states in $\langle D\rangle$ is

$$
\begin{aligned}
A^{c}(- & \left.A^{2}-A^{-2}\right)^{v-1}+\sum_{i=1}^{e} \sum_{j=1}^{k_{i}}\left(\begin{array}{c}
k_{i} \\
j
\end{array}\right) A^{c-2 j}\left(-A^{2}-A^{-2}\right)^{v-2+j-1} \\
= & (-1)^{v-1}\left(A^{c+2 v-2}+(v-1) A^{c+2 v-6}+\left(\begin{array}{c}
v-1 \\
2
\end{array}\right) A^{c+2 v-10}+\cdots\right) \\
& +\sum_{i=1}^{e} \sum_{j=1}^{k_{i}}\left(\begin{array}{c}
k_{i} \\
j
\end{array}\right)(-1)^{v+j-3} A^{c+2 v-6}+\sum_{i=1}^{e} \sum_{j=1}^{k_{i}}\left(\begin{array}{c}
k_{i} \\
j
\end{array}\right)(-1)^{v+j-3}(v+j-3) A^{c+2 v-10}+\cdots \\
= & (-1)^{v-1} A^{c+2 v-2}+(-1)^{v-2}(e-v+1) A^{c+2 v-6} \\
& +(-1)^{v-3}\left(\left(\begin{array}{c}
v-1 \\
2
\end{array}\right)-e(v-2)+\mu\right) A^{c+2 v-10}+\text { lower degree terms. }
\end{aligned}
$$

The last equation follows from

$$
\sum_{j=1}^{k_{i}}\left(\begin{array}{c}
k_{i} \\
j
\end{array}\right)(-1)^{j} j=\left\{\begin{aligned}
-1 & k_{i}=1 \\
0 & k_{i}>1
\end{aligned}\right.
$$

Case 2. States whose $B$-splicings appear in a pair of distinct multiple edges, which are either disjoint or separated. The total contribution of such states in $\langle D\rangle$ is

$$
\begin{gathered}
\sum_{i=1}^{k_{r}} \sum_{j=1}^{k_{s}}\left(\begin{array}{c}
k_{r} \\
i
\end{array}\right)\left(\begin{array}{c}
k_{s} \\
j
\end{array}\right) A^{c-2(i+j)}\left(-A^{2}-A^{-2}\right)^{v-4+i+j-1} \\
=(-1)^{v-3} A^{c+2 v-10}+\text { lower order terms. }
\end{gathered}
$$

Case 3. States whose $B$-splicings appear in a pair of distinct multiple edges $E, E^{\prime}$ which are mixed at a vertex. This is the most delicate of the four cases. We claim that there is no contribution to the three leading coefficients that involve $B$-splicings at both edges.

Suppose the $A$-diagram is locally similar to Figure 4, i.e.

$$
D=D\left(p_{1}, q_{1}, p_{2}, q_{2}, \ldots, p_{k}, q_{k}\right),
$$

with $p_{i}>0$ and $q_{i}>0$ for $i=1, \ldots, k$. We will deal with the following subcases.

(i) One of the $p_{i}$ or one of the $q_{i}$ is greater than 1 . Assume that $p_{1}>1$. In this case one can see that the three leading coefficients of $\left\langle D\left(p_{1}, q_{1}, \ldots, p_{k}, q_{k}\right)\right\rangle$ equal the three leading coefficients of $\left\langle D\left(p_{1}-1, q_{1}, \ldots, p_{k}, q_{k}\right)\right\rangle$. Therefore, to show the claim we can assume that $p_{1}=\cdots=p_{k}=$ $q_{1}=\cdots=q_{k}=1$.

(ii) If $k=2$, i.e. our diagram is $D(1,1,1,1)$, a straightforward computation shows that the contribution of the type of $B$-splicings we consider here vanishes.

(iii) We are left with the case of $k>2$. We compare the leading terms of

$$
\left\langle D\left(p_{1}=1, q_{1}=1, p_{2}=1, q_{2}=1, \ldots, p_{k}=1, q_{k}=1\right)\right\rangle
$$

and

$$
\left\langle D\left(p_{1}=0, q_{1}=1, p_{2}=2, q_{2}=1, \ldots, p_{k}=1, q_{k}=1\right)\right\rangle=\left\langle D\left(p_{2}=2, q_{2}=1, \ldots, p_{k}=1, q_{k}=2\right)\right\rangle .
$$

Yet another direct, straightforward computation shows that the three leading coefficients coincide. Thus we can reduce this case to (i) and (ii). 


\section{COLORED JONES POLYNOMial}

Thus we showed that the sum of all states with $B$-splicings at a pair of mixed edges does not contribute to the leading three coefficients. Recall that in our notation $\theta$ is the number of pairs of edges in $A(D)^{\prime}$ that are mixed. To summarize Case 2 and Case 3: the total contribution of states whose $B$-splicings appear in a pair of distinct multiple edges is

$$
(-1)^{v-3}\left(\left(\begin{array}{l}
e \\
2
\end{array}\right)-\theta\right) A^{c+2 v-10}+\text { lower order terms. }
$$

Case 4. States with three $B$-splicings appearing on three different edges in $A(D)^{\prime}$ that form a triangle. Note that for reasons of planarity the edges cannot be mixed at any of the three vertices.

The total contribution of such states is

$$
\begin{aligned}
& \sum_{\tau} \sum_{i=1}^{k_{r}} \sum_{j=1}^{k_{s}} \sum_{k=1}^{k_{t}}\left(\begin{array}{c}
k_{r} \\
i
\end{array}\right)\left(\begin{array}{c}
k_{s} \\
j
\end{array}\right)\left(\begin{array}{c}
k_{t} \\
k
\end{array}\right) A^{c-2(i+j+k)}\left(-A^{2}-A^{-2}\right)^{v-1+i+j+k-3-1} \\
& =(-1)^{v-3}(-\tau) A^{c+2 v-10}+\text { lower order terms. }
\end{aligned}
$$

This proves the theorem.

Similar to the discussion in $\S 3$, we need to compare first the third highest degree of $\left\langle D^{n}\right\rangle$ and the highest degree of $\left\langle D^{n-2}\right\rangle$ :

$$
\left(n^{2} c+2 n v-10\right)-\left((n-2)^{2} c+2(n-2) v-2\right)=(4 n-4) c+4 v-8>0,
$$

since we have $v \geqslant 2$ and $n \geqslant 2$ for non-trivial cases. Thus, we just need to consider the third leading coefficient of $\left\langle D^{n}\right\rangle$.

Denote by $v_{n}$ the number of vertices of $A\left(D^{n}\right)^{\prime}, e_{n}$ the number of edges in $A\left(D^{n}\right)^{\prime}, \mu_{n}$ the number of edges in $A\left(D^{n}\right)$ whose multiplicity in $A\left(D^{n}\right)$ is larger than $1, \theta_{n}$ the number of pairs of edges in $A\left(D^{n}\right)^{\prime}$ mixed at a vertex, and $\tau_{n}$ the number of triangles in $A\left(D^{n}\right)^{\prime}$.

Lemma 4.2. Suppose the $A$-adequate diagram $D$ contains no kinks, or $A(D)$ has no vertices of valence 1. We have $v_{n}=n v, e_{n}=e+(n-1) v, \mu_{n}=e_{n}=e+(n-1) v$ when $n>1$, and $\tau_{n}=\tau$. Furthermore, if $D$ is a reduced alternating knot diagram and $n>1$, then $\theta_{n}=(n-2) v+2 e$.

Proof. This is clear from the structure of $A\left(D^{n}\right)^{\prime}$.

The relevant terms in Theorem 4.1 for the third coefficient are now simplified with the following lemma.

Lemma 4.3. If $D$ is a reduced alternating knot diagram and $n>1$, we have

$$
\left(\begin{array}{c}
v_{n}-1 \\
2
\end{array}\right)-e_{n}\left(v_{n}-2\right)+\mu_{n}+\left(\begin{array}{c}
e_{n} \\
2
\end{array}\right)-\theta_{n}-\tau_{n}=\frac{(e-v)^{2}+(e-v)}{2}-\tau+1 .
$$

Thus, we have finally arrived at our main theorem.

Theorem 4.4. Let $K$ be an alternating knot. Write

$$
\begin{aligned}
J_{K}^{\prime}(n)= & \pm\left(a_{n} A^{k_{n}}-b_{n} A^{k_{n}-4}+c_{n} A^{k_{n}-8}\right) \pm \cdots \\
& \pm\left(\gamma_{n} A^{k_{n}-4 r_{n}+8}-\beta_{n} A^{k_{n}-4 r_{n}+4}+\alpha_{n} A^{k_{n}-4 r_{n}}\right)
\end{aligned}
$$

with positive $a_{n}$ and $\alpha_{n}$.

Let $A(D)$ and $B(D)$ be the $A$ - and $B$-graphs of a reduced alternating diagram $D$ of $K$ with crossing number $c$. The reduced graphs $A(D)^{\prime}$ and $B(D)^{\prime}$ have $e_{A}$ and $e_{B}$ edges and $v_{A}$ and $v_{B}$ vertices. Note that $v_{A}+v_{B}=c+2$. Furthermore, there are $\tau_{A}$ and $\tau_{B}$ triangles in $A(D)^{\prime}$ and $B(D)^{\prime}$. Then: 


\section{O. T. DAsbach And X.-S. Lin}

(i) the span $r_{n}$ of $J_{K}^{\prime}(n)$, i.e. the difference of the highest and lowest exponent, in the variable $q=A^{4}$, is

$$
r_{n}=\left(\begin{array}{l}
n \\
2
\end{array}\right) c
$$

(ii) $a_{n}=\alpha_{n}=1$;

(iii) $b_{n}=e_{A}-v_{A}+1$ and $\beta_{n}=e_{B}-v_{B}+1$;

(iv)

$$
c_{n}=\left(\begin{array}{c}
b_{n} \\
2
\end{array}\right)-\tau_{A} \quad \text { and } \quad \gamma_{n}=\left(\begin{array}{c}
\beta_{n} \\
2
\end{array}\right)-\tau_{B}
$$

for $n>2$, the coefficients $c_{2}$ and $\gamma_{2}$ having been identified in [DL04] as

$$
\begin{aligned}
& c_{2}=\left(\begin{array}{c}
b_{2}+1 \\
2
\end{array}\right)+n(2)-\tau_{A}, \\
& \gamma_{2}=\left(\begin{array}{c}
\beta_{2}+1 \\
2
\end{array}\right)+n^{*}(2)-\tau_{B},
\end{aligned}
$$

where $n(2)$ (or $n^{*}(2)$ ) is the number of edges in $A^{\prime}(D)$ (or $B^{\prime}(D)$ ) that correspond to edges of multiplicity at least 2 in the unreduced graph $A(D)$ (or $B(D)$ ).

In particular, $\left|a_{n}\right|,\left|b_{n}\right|,\left|\alpha_{n}\right|$ and $\left|\beta_{n}\right|$ are independent of $n$ when $n>1$ and $\left|c_{n}\right|$ and $\left|\gamma_{n}\right|$ are independent of $n$ when $n>2$.

Proof. Item (i) was essentially shown by Kurpita and Murasugi in [KM92] and Le in [Le04]. The highest degree of $\left\langle S\left(D^{n}\right)\right\rangle$ in $A$ is $n^{2} c+2 n v_{a}-2$ and the lowest degree is $-n^{2} c-2 n v_{b}+2$. Thus for the span of $\left\langle S\left(D^{n}\right)\right\rangle$ in the variable $A$ we get

$$
2 n^{2} c+2 n\left(v_{A}+v_{B}\right)+4=2 n^{2} c+2 n(c+2)+4 .
$$

This implies that the span of $J_{K}(n+1)$ is

$$
2 n^{2} c+2 n(c+2)
$$

and thus the span of $J_{K}^{\prime}(n+1)$ in the variable $A$ is

$$
2 n^{2} c+2 n(c+2)-4 n=2 n^{2} c+2 n c .
$$

Changing to the variable $q$ this shows claim (i).

For the remaining claims: recall that we showed

$$
\begin{aligned}
(-1)^{p_{n}} A^{l_{n}}\left\langle S\left(D^{n}\right)\right\rangle= & 1-\left(e_{A}-v_{A}+1\right) A^{-4} \\
& +\left(\frac{\left(e_{A}-v_{A}\right)^{2}+\left(e_{A}-v_{A}\right)}{2}-\tau_{A}+1\right) A^{-8} \\
& + \text { lower order terms }
\end{aligned}
$$

for some numbers $p_{n}$ and $l_{n}$.

The remaining claims now follow from a comparison of the coefficients of $\left\langle S\left(D^{n}\right)\right\rangle$ and $J_{K}^{\prime}(n+1)$ and the fact that the $A$-graph of the knot is the $B$-graph of the mirror image of the knot and vice versa.

\section{Example}

As an example we pick the alternating knots $12_{217}$ and $12_{1228}$ in the Knotscape census [HTW98]. The two knots are given in Figure 5. According to Knotscape both knots share the same Jones polynomial $J_{K}^{\prime}(2)$. 


\section{COLORED JONES POLYNOMIAL}
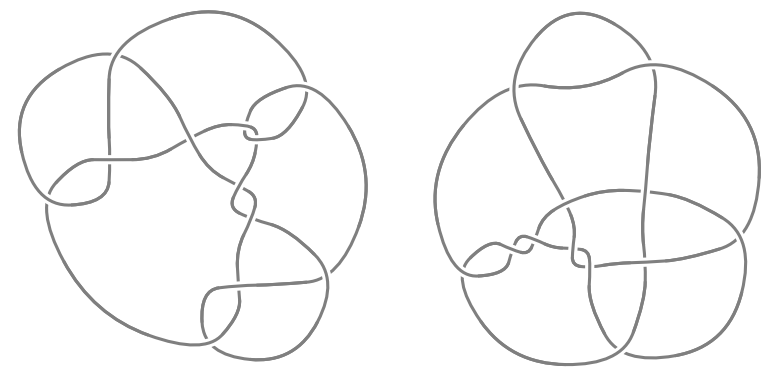

FiguRE 5. The alternating knots $12_{217}$ and $12_{1228}$.

We have the following for the knot $12_{217}$ : the $A$-graph is the checkerboard graph corresponding to the color not containing the outer face. Its reduced graph has $e_{A}=9$ edges and $v_{A}=6$ vertices. Furthermore, it contains $\tau_{A}=4$ triangles. Thus the three leading coefficients are, up to a common sign change, $1,-\left(e_{A}-v_{A}+1\right)=-4$ and $\left(\begin{array}{l}4 \\ 2\end{array}\right)-\tau_{A}=2$. Similarly, $e_{B}=11, v_{B}=8$ and $\tau_{B}=2$. Thus the last three coefficients are $4,-4,1$, up to a common sign.

For the knot 121228 the data are $e_{A}=9, v_{A}=6, \tau_{A}=3, e_{B}=11, v_{B}=8, \tau_{B}=2$ and we get for the leading coefficients $1,-4,3$ and for the last three coefficients $4,-4,1$. Again, these two lists are up to sign changes.

Since the Jones polynomials of the two knots coincide but already the third coefficient of the colored Jones polynomials for color $n>2$ does not, we see the following lemma.

Lemma 5.1. For $n>2$ the leading third coefficient of the colored Jones polynomial $J_{K}^{\prime}(n)$ is in general not determined by the Jones polynomial $J_{K}^{\prime}(2)$.

\section{The volume-ish theorem for the colored Jones polynomial}

As a corollary to the main theorem (Theorem 4.4) and the results in [DL04] we also get the volumeish theorem for the colored Jones polynomial.

Theorem 6.1 (Volume-ish theorem for the colored Jones polynomial). For an alternating, prime, non-torus knot $K$ let

$$
J_{K}^{\prime}(n)=a_{n} q^{k_{n}}+b_{n} q^{k_{n}-1}+\cdots+\beta_{n} q^{k_{n}-r_{n}+1}+\alpha_{n} q^{k_{n}-r_{n}}
$$

be the colored Jones polynomial.

Then with $b:=b_{2}$ and $\beta:=\beta_{2}$ we have $b=b_{n}$ and $\beta=\beta_{n}$ for all $n$ and

$$
2 v_{0}(\max (|b|,|\beta|)-1) \leqslant \operatorname{Vol}\left(S^{3}-K\right) \leqslant 10 v_{0}(|b|+|\beta|-1),
$$

where $v_{0} \approx 1.0149416$ is the volume of an ideal regular hyperbolic tetrahedron.

\section{ACKNowledgements}

We are more than indebted to Dror Bar-Natan for making his wonderful Mathematica package KnotTheory publicly available on his Web page [BN06]. The colored Jones polynomial routine allowed us to experimentally observe some of the phenomena that we were able to show here. 


\section{COLORED Jones POLYNOMial}

\section{REFERENCES}

BN06 D. Bar-Natan, KNotTheory (a Mathematica package), available at http://www.math.toronto.edu/ drorbn/.

DL04 O. T. Dasbach and X.-S. Lin, A volume-ish theorem for the Jones polynomial of alternating knots, Preprint (2004), math.GT/0403448.

HTW98 J. Hoste, M. Thistlethwaite and J. Weeks, The first 1,701,936 knots, Math. Intelligencer 20 (1998), no. $4,33-48$.

Kas97 R. M. Kashaev, The hyperbolic volume of knots from the quantum dilogarithm, Lett. Math. Phys. 39 (1997), 269-275.

KM92 B. I. Kurpita and K. Murasugi, On a hierarchal Jones invariant, in Knots 90, Osaka, 1990 (de Gruyter, Berlin, 1992), 489-542.

Lac04 M. Lackenby, The volume of hyperbolic alternating link complements, with an appendix by I. Agol and D. Thurston, Proc. London Math. Soc. (3) 88 (2004), 204-224.

Le04 T. T. Q. Le, The colored Jones polynomial and the A-polynomial of knots, Preprint (2004), math.GT/0407521.

Lic97 W. B. R. Lickorish, An introduction to knot theory, Graduate Texts in Mathematics, vol. 175 (Springer, New York, 1997).

MM01 H. Murakami and J. Murakami, The colored Jones polynomials and the simplicial volume of a knot, Acta Math. 186 (2001), 85-104.

Sto04 A. Stoimenow, The second coefficient of the Jones polynomial, in Proc. conf. 'Intelligence of Low Dimensional Topology 2004' (Osaka City University, Osaka, 2004).

Thi88 M. B. Thistlethwaite, On the Kauffman polynomial of an adequate link, Invent. Math. 93 (1988), $285-296$.

Oliver T. Dasbach kasten@math.Isu.edu

Department of Mathematics, Louisiana State University, Baton Rouge, LA 70803, USA

Xiao-Song Lin $x \mid @ m a t h . u c r . e d u$

Department of Mathematics, University of California, Riverside, CA 92521, USA 